\title{
On conformally doubly warped product Finsler manifold
}

\author{
A. Soleiman ${ }^{1,2^{*}}$ and A.M. Abdelsalam ${ }^{1}$
}

\section{${ }^{*}$ Correspondence:}

amrsoleiman@yahoo.com 'Department of Mathematics, Faculty of Sciences, Benha University, Benha, Egypt ${ }^{2}$ Department of Mathematics, Collage of Sciences and Arts in Gurayat Jouf University, Skaka, Kingdom of Saudi Arabia

\begin{abstract}
The aim of the present paper is to introduce the notion of conformally doubly warped product Finsler manifold (CDWPF). For such a Finsler manifold, the coefficients of Barthel connection and its curvature tensor are investigated. The coefficients of Cartan, Berwald, Hashiguchi and Chern (Rund) connections of CDWPF are calculated. Some special Finsler spaces are studied.

Keywords: Finsler manifold; Barthel connection; Cartan connection; Berwald connection; Conformally doubly warped product Finsler manifold; Riemannian spaces; C-reducible spaces; Berwald spaces
\end{abstract}

MSC 2010: 53C60, 53B40, 58B20

\section{Introduction}

The doubly warped product of Riemannian (semi-Riemannian) manifolds has been studied by many authors, for example, we refer to [1-5]. Several applications to theoretical physics can be found in the literature. For instance, in [3], Beem-Powell considered the doubly warped product for Lorentzian manifolds. Moreover, in [6, 7], Asanov studied the generalization of the Schwarzschild metric in the Finslerian setting and obtained some models of relativity theory described through the warped product of Finsler metrics. Then, Shen used a construction of warped of Riemannian metrics at the vertical bundle and obtained a Finslerian warped product metric [8]. Recently, E. Peyghan and A. Tayebi ([9]) introduced horizontal and vertical warped product Finsler manifold and they proved that every C-reducible or proper Berwaldian doubly warped product Finsler manifold is Riemannian.

In this paper, we study a more general product Finsler manifold that will be called conformally doubly warped product Finsler manifold (CDWPF), that is, the product manifold $M:=M_{1} \times M_{2}$ endowed with the metric $F: T M_{1}^{o} \times T M_{2}^{o} \rightarrow R$ defined by

$$
F\left(v_{1}, v_{2}\right)=e^{\sigma\left(\pi_{1}\left(v_{1}\right), \pi_{2}\left(v_{2}\right)\right)} \sqrt{f_{2}^{2}\left(\pi_{2}\left(v_{2}\right)\right) F_{1}^{2}\left(\pi_{1}\left(v_{1}\right)\right)+f_{1}^{2}\left(\pi_{1}\left(v_{1}\right)\right) F_{2}^{2}\left(\pi_{2}\left(v_{2}\right)\right)},
$$

where $\left(M_{1}, F_{1}\right)$ and $\left(M_{2}, F_{2}\right)$ are two Finsler manifolds; $f_{1}: M_{1} \rightarrow R^{+}$and $f_{2}: M_{2} \rightarrow R^{+}$ are two smooth functions on $M_{1}$ and $M_{2}$, respectively; $\pi_{1}: M_{1} \times M_{2} \rightarrow M_{1}, \pi_{2}: M_{1} \times$ $M_{2} \rightarrow M_{2}$ are the natural projection maps; and $\sigma: M_{1} \times M_{2} \rightarrow R^{+}$is a positively smooth function on $M_{1} \times M_{2}, T M_{1}^{o}=T M_{1}-0$ and $T M_{2}^{o}=T M_{2}-0$.

For a conformally doubly warped product Finsler manifolds (CDWPF), the coefficients of Barthel connection and its curvature tensor are studied. Moreover, the coefficients of

(c) The Author(s). 2019 Open Access This article is distributed under the terms of the Creative Commons Attribution 4.0 International License (http://creativecommons.org/licenses/by/4.0/), which permits unrestricted use, distribution, and reproduction in any medium, provided you give appropriate credit to the original author(s) and the source, provide a link to the Creative Commons license, and indicate if changes were made. 
Cartan connection for CDWPF are given. Finally, some special Finsler spaces concerning a conformally doubly warped product Finsler manifold are derived.

Finally, the obtained results in this paper generalize and retrieve some results concerning the doubly warped product Finsler manifold, warped product Finsler manifold, conformally warped product Finsler manifold, product Finsler manifold and conformally product Finsler manifold.

\section{Notations and preliminaries}

In this section, we give a brief account of the basic concepts of Finsler geometry that will be needed throughout. This means that all notations and results which appear in this section refer to [10-14].

Let $M$ be an $n$-dimensional smooth manifold. Let $\left(x^{i}\right)$ be the coordinates of any point of the base manifold $M$ and $\left(y^{i}\right)$ a supporting element at the same point. We mean by $T_{x} M$ the tangent space at $x \in M$ and $T M=\bigcup_{x \in M} T_{x} M$ the tangent bundle of $M$. A Finsler structure on $M$ is defined as follows:

Definition 1 A Finsler structure on a manifold $M$ is a function [11-13]

$$
F: T M \rightarrow \mathbb{R}
$$

with the following properties:

(a) $F \geqslant 0$ and $F(x, y)=0$ if and only if $y=0$.

(b) $F$ is $C^{\infty}$ on the slit tangent bundle $\mathcal{T} M:=T M \backslash\{0\}$.

(c) $F(x, y)$ is positively homogenous of degree one in $y: F(x, \lambda y)=\lambda F(x, y)$ for all $y \in T M$ and $\lambda>0$.

(d) The Hessian matrix $g_{i j}(x, y):=\frac{1}{2} \frac{\partial^{2} F^{2}}{\partial y^{j} \partial y^{i}}$ is positive-definite at each point of $\mathcal{T} M$.

The pair $(M, F)$ is called a Finsler space and the symmetric bilinear form $g=g_{i j}(x, y) d x^{i} \otimes$ $d x^{j}$ is called the Finsler metric tensor of the Finsler space $(M, F)$.

The tensor $C_{i j k}:=\frac{1}{4} \frac{\partial^{3} F^{2}}{\partial y^{k} \partial y^{j} \partial y^{i}}$ is called the Cartan torsion. It is well known that $F$ is called Riemannian if and only if the Cartan tensor $C_{i j k}$ vanishes identically $[10,13]$. By Deicke's Theorem, $F$ is Riemannian if and only if $I_{i}=0$, where $I_{i}:=g^{j k} C_{i j k}$ called the mean (contraction) Cartan torsion.

The Matsumoto torsion for a Finsler structure $(M, F)$ with dimension $n$ is given by

$$
M_{i j k}:=C_{i j k}+\frac{1}{n+1}\left\{I_{i} \hbar_{j k}+I_{j} \hbar_{k i}+I_{k} \hbar_{i j}\right\}
$$

where $\hbar_{i j}:=g_{i j}-\frac{1}{F^{2}} y_{i} y_{j}$ is the angular metric tensor and $y_{i}:=g_{i j} y^{j}=\frac{\partial F}{\partial y^{i}}$. A Finsler structure $F$ is said to be C-reducible if the Matsumoto torsion vanishes identically [15].

If a Finsler manifold $(M, F(x, y))$ is given, then the components of the associated canonical spray $G^{h}$ and the components of the associated nonlinear connection (Barthel connection) $G_{i}^{h}$ are defined respectively by

$$
G^{i}(x, y):=\frac{1}{4} g^{i h}\left\{\frac{\partial^{2} F^{2}}{\partial y^{h} \partial x^{j}} y^{j}-\frac{\partial F^{2}}{\partial x^{h}}\right\}(x, y), G_{i}^{h}:=\frac{\partial G^{h}}{\partial y^{i}} .
$$

Also, the Berwald curvature tensor is defined by

$$
B_{i j k}^{h}:=\frac{\partial^{3} G^{h}}{\partial y^{k} \partial y^{j} \partial y^{i}} .
$$


A Finsler structure $F$ is called Berwaldian if the Berwald curvature tensor $B_{i j k}^{h}$ vanishes identically.

Finally, we know that there exist at least four linear Finsler connections associated with a Finsler structure $F$ and they have the same nonlinear connection $G_{i}^{h}$ namely, the Cartan connection $C \Gamma \equiv\left(\Gamma_{i j}^{h}, G_{i}^{h}, C_{i j}^{h}\right)$, the Berwald connection $B \Gamma \equiv\left(G_{i j}^{h}, G_{i}^{h}, 0\right)$, the Hashiguchi connection $H \Gamma \equiv\left(G_{i j}^{h}, G_{i}^{h}, C_{i j}^{h}\right)$, and the Chern (Rund) connection $R \Gamma \equiv\left(\Gamma_{i j}^{h}, G_{i}^{h}, 0\right)$, where $C_{i j}^{h}:=\frac{1}{2} g^{h \ell} \frac{\partial g_{\ell j}}{\partial y^{i}}, G_{i j}^{h}:=\frac{\partial G_{i}^{h}}{\partial y^{j}}$ and $\Gamma_{i j}^{k}:=\frac{1}{2} g^{k h}\left\{\frac{\delta g_{h j}}{\delta x^{i}}+\frac{\delta g_{h i}}{\delta x^{i}}-\frac{\delta g_{i j}}{\delta x^{h}}\right\}$; $\frac{\delta}{\delta x^{k}}:=\frac{\partial}{\partial x^{k}}-G_{k}^{m} \frac{\partial}{\partial y^{m}}$ being the horizontal basis adapted to the Barthel connection $G_{j}^{i}$.

\section{Barthel connection for CDWPF}

In this section, we investigate the coefficients of canonical spray for the conformally doubly warped product Finsler manifold (CDWPF). Moreover, the coefficients of the Barthel connection and its curvature tensor for CDWPF are obtained.

First, we begin with the following definition.

Definition 2 Let $\left(M_{1}, F_{1}\right)$ and $\left(M_{2}, F_{2}\right)$ be two Finsler manifolds with dimM $M_{1}=n_{1}$ and $\operatorname{dim} M_{2}=n_{2}$ and $f_{1}: M_{1} \rightarrow R^{+}$and $f_{2}: M_{2} \rightarrow R^{+}$be two smooth functions. Let $\pi_{1}: M_{1} \times M_{2} \rightarrow M_{1}, \pi_{2}: M_{1} \times M_{2} \rightarrow M_{2}$ be the natural projection maps and $\sigma: M_{1} \times M_{2} \rightarrow R^{+}$be positively smooth function on $M_{1} \times M_{2}$. The product manifold $M:=M_{1} \times M_{2}$ endowed with the metric $F: T M_{1}^{o} \times T M_{2}^{o} \rightarrow R$ defined by

$$
F\left(v_{1}, v_{2}\right)=e^{\sigma\left(\pi_{1}\left(v_{1}\right), \pi_{2}\left(v_{2}\right)\right)} \sqrt{f_{2}^{2}\left(\pi_{2}\left(v_{2}\right)\right) F_{1}^{2}\left(\pi_{1}\left(v_{1}\right)\right)+f_{1}^{2}\left(\pi_{1}\left(v_{1}\right)\right) F_{2}^{2}\left(\pi_{2}\left(v_{2}\right)\right)},
$$

where $T M_{1}^{o}=T M_{1}-0$ and $T M_{2}^{o}=T M_{2}-0$, called the conformally doubly warped product Finsler manifolds (CDWPF) of the manifolds $M_{1}$ and $M_{2}$, and denoted by $\left(f_{2} M_{1} \times f_{1} M_{2}, F\right)$. In this case, $\sigma$ will be called the conformally factor and $f_{1}$ and $f_{2}$ will be called the warping functions.

Specially, if either $f_{1}=1$ or $f_{2}=1$, but not both, and $\sigma$ is not constant function, then $\left(f_{2} M_{1} \times f_{1} M_{2}, F\right)$ will be called conformally warped product Finsler manifolds (CWPF) of the manifolds $M_{1}$ and $M_{2}$. If both $f_{1}=1, f_{2}=1$, and $\sigma$ is not constant function, then $\left(f_{2} M_{1} \times{ }_{f_{1}} M_{2}, F\right)$ will be called a conformally product Finsler manifold (CPF). If neither $f_{1}$ nor $f_{2}$ is constant and $\sigma=0$, then $\left(f_{2} M_{1} \times{ }_{f_{1}} M_{2}, F\right)$ will be called a doubly warped product Finsler manifold (DWPF).

Now, let $\left(M_{1}, F_{1}\right)$ and $\left(M_{2}, F_{2}\right)$ be two Finsler manifolds with dimensions $n_{1}$ and $n_{2}$, respectively. Hence, the two functions

$$
g_{i j}(x, y):=\frac{\partial^{2} F_{1}^{2}}{\partial y^{i} \partial y^{j}} \quad g_{\alpha \beta}(u, v):=\frac{\partial^{2} F_{2}^{2}}{\partial \nu^{\alpha} \partial v^{\beta}}
$$

define Finsler metrics on $M_{1}$ and $M_{2}$, respectively. Let $\left({ }_{f_{2}} M_{1} \times{ }_{f_{1}} M_{2}, F\right)$ be a conformally doubly warped Finsler manifold (CDWPF) and let $\mathrm{x} \in M$ and $\mathrm{y} \in T_{\mathrm{x}} M$, where $\mathrm{x}=$ $(x, u), \mathrm{y}=(y, v), M:=M_{1} \times M_{2}$ and $T_{\mathrm{x}} M=T_{x} M_{1} \oplus T_{u} M_{2}$. 
Consequently, from Eqs. (1) and (2), the conformally doubly warped Finsler metric and its inverse are given by

$$
\begin{aligned}
& \mathbf{g}_{a b}(\mathrm{x}, \mathrm{y}):=\frac{\partial^{2} F^{2}(\mathrm{x}, \mathrm{y})}{\partial \mathrm{y}^{a} \partial \mathrm{y}^{b}}=\left(\begin{array}{cc}
e^{2 \sigma(x, u)} f_{2}^{2} g_{i j} & 0 \\
0 & e^{2 \sigma(x, u)} f_{1}^{2} g_{\alpha \beta}
\end{array}\right) \\
& \mathbf{g}^{a b}(\mathrm{x}, \mathrm{y})=\left(\begin{array}{cc}
e^{-2 \sigma(x, u)} \frac{1}{f_{2}^{2}} g^{i j} & 0 \\
0 & e^{-2 \sigma(x, u)} \frac{1}{f_{1}^{2}} g^{\alpha \beta}
\end{array}\right),
\end{aligned}
$$

where $\mathrm{y}^{a}:=\left(y^{i}, v^{\alpha}\right), \mathrm{y}^{b}:=\left(y^{j}, v^{\beta}\right), \quad \mathbf{g}_{i j}=e^{2 \sigma(x, u)} f_{2}^{2} g_{i j}, \mathbf{g}_{\alpha \beta}=e^{2 \sigma(x, u)} f_{1}^{2} g_{\alpha \beta}, \mathbf{g}_{i \beta}=$ $\mathbf{g}_{\alpha j}=0 ; i, j, \ldots \in\left\{1, \ldots, n_{1}\right\}, \alpha, \beta, \ldots \in\left\{1, \ldots, n_{2}\right\}$ and $a, b, \ldots \in\left\{1, \ldots, n_{1}+n_{2}\right\}$.

Proposition 1 The coefficients of conformally doubly warped canonical spray for CDWPF are given by

$$
\mathbb{G}^{a}(x, u, y, v)=\left(\mathbb{G}^{i}(x, u, y, v), \mathbb{G}^{\alpha}(x, u, y, v)\right),
$$

where

$$
\begin{aligned}
\mathbb{G}^{i}(x, u, y, v)= & G^{i}(x, y)+\frac{1}{4} g^{i h}\left\{2\left(\frac{\partial \sigma}{\partial x^{j}} y^{j}+\frac{\partial \sigma}{\partial u^{\alpha}} \nu^{\alpha}\right) \frac{\partial F_{1}^{2}}{\partial y^{h}}-\frac{1}{f_{2}^{2}} \frac{\partial \sigma}{\partial x^{h}}\left(f_{2}^{2} F_{1}^{2}+f_{1}^{2} F_{2}^{2}\right)\right. \\
& \left.+\frac{1}{f_{2}^{2}}\left(\frac{\partial f_{2}^{2}}{\partial u^{\alpha}} \frac{\partial F_{1}^{2}}{\partial y^{h}} v^{\alpha}-\frac{\partial f_{1}^{2}}{\partial x^{h}} F_{2}^{2}\right)\right\} \\
\mathbb{G}^{\alpha}(x, u, y, v)= & G^{\alpha}(u, v)+\frac{1}{4} g^{\alpha \gamma}\left\{2\left(\frac{\partial \sigma}{\partial u^{\beta}} \nu^{\beta}+\frac{\partial \sigma}{\partial x^{j}} y^{j}\right) \frac{\partial F_{2}^{2}}{\partial \nu^{\alpha}}-\frac{1}{f_{1}^{2}} \frac{\partial \sigma}{\partial u^{\gamma}}\left(f_{2}^{2} F_{1}^{2}+f_{1}^{2} F_{2}^{2}\right)\right. \\
& \left.+\frac{1}{f_{1}^{2}}\left(\frac{\partial f_{1}^{2}}{\partial x^{j}} \frac{\partial F_{2}^{2}}{\partial \nu^{\beta}} y^{j}-\frac{\partial f_{2}^{2}}{\partial u^{\gamma}} F_{1}^{2}\right)\right\} .
\end{aligned}
$$

Proof We know that the coefficients of canonical spray for $\left(M_{1}, F_{1}\right),\left(M_{2}, F_{2}\right)$, and $\left(f_{2} M_{1} \times f_{1} M_{2}, F\right)$ are defined respectively by

$$
\begin{aligned}
G^{i}(x, y) & =\frac{1}{4} g^{i h}\left\{\frac{\partial^{2} F_{1}^{2}}{\partial y^{h} \partial x^{j}} y^{j}-\frac{\partial F_{1}^{2}}{\partial x^{h}}\right\}(x, y) \\
G^{\alpha}(u, v) & =\frac{1}{4} g^{\alpha \gamma}\left\{\frac{\partial^{2} F_{2}^{2}}{\partial v^{\gamma} \partial u^{\beta}} v^{\beta}-\frac{\partial F_{2}^{2}}{\partial u^{\gamma}}\right\}(u, v) \\
\mathbb{G}^{a}(\mathrm{x}, \mathrm{y}) & =\frac{1}{4} \mathbf{g}^{a b}\left\{\frac{\partial^{2} F^{2}}{\partial \mathrm{y}^{b} \partial \mathrm{x}^{c}} \mathrm{y}^{c}-\frac{\partial F^{2}}{\partial \mathrm{x}^{b}}\right\}(\mathrm{x}, \mathrm{y}) .
\end{aligned}
$$

Setting $a=i$ into (9) and noting the fact that $\mathbf{g}^{i \beta}=0$, we get

$$
\begin{aligned}
\mathbb{G}^{i}(\mathrm{x}, \mathrm{y}) & =\frac{1}{4} \mathbf{g}^{i b}\left\{\frac{\partial^{2} F^{2}}{\partial \mathrm{y}^{b} \partial \mathrm{x}^{c}} \mathrm{y}^{c}-\frac{\partial F^{2}}{\partial \mathrm{x}^{b}}\right\}(\mathrm{x}, \mathrm{y}) \\
& =\frac{1}{4} \mathbf{g}^{i h}\left\{\frac{\partial^{2} F^{2}}{\partial y^{h} \partial x^{j}} y^{j}+\frac{\partial^{2} F^{2}}{\partial y^{h} \partial u^{\alpha}} \nu^{\alpha}-\frac{\partial F^{2}}{\partial x^{h}}\right\} .
\end{aligned}
$$


On the other hand, from (1) one can show that

$$
\begin{aligned}
\frac{\partial F^{2}}{\partial x^{j}} & =e^{2 \sigma}\left\{2 \frac{\partial \sigma}{\partial x^{j}}\left(f_{2}^{2} F_{1}^{2}+f_{1}^{2} F_{2}^{2}\right)+f_{2}^{2} \frac{\partial F_{1}^{2}}{\partial x^{j}}+\frac{\partial f_{1}^{2}}{\partial x^{j}} F_{2}^{2}\right\} \\
\frac{\partial F^{2}}{\partial u^{\alpha}} & =e^{2 \sigma}\left\{2 \frac{\partial \sigma}{\partial u^{\alpha}}\left(f_{2}^{2} F_{1}^{2}+f_{1}^{2} F_{2}^{2}\right)+f_{1}^{2} \frac{\partial F_{2}^{2}}{\partial u^{\alpha}}+\frac{\partial f_{2}^{2}}{\partial u^{\alpha}} F_{1}^{2}\right\} \\
\frac{\partial^{2} F^{2}}{\partial y^{h} \partial x^{j}} & =e^{2 \sigma} f_{2}^{2}\left\{2 \frac{\partial \sigma}{\partial x^{j}} \frac{\partial^{2} F_{1}^{2}}{\partial y^{h}}+\frac{\partial^{2} F_{1}^{2}}{\partial y^{h} \partial x^{j}}\right\} \\
\frac{\partial^{2} F^{2}}{\partial y^{h} \partial u^{\alpha}} & =e^{2 \sigma}\left\{2 f_{2}^{2} \frac{\partial \sigma}{\partial u^{\alpha}} \frac{\partial^{2} F_{1}^{2}}{\partial y^{h}}+\frac{\partial f_{2}^{2}}{\partial u^{\alpha}} \frac{\partial F_{1}^{2}}{\partial y^{h}}\right\} .
\end{aligned}
$$

Hence, Relation (5) follows by substituting the above relations into (10), taking into account (3), (4) and (7).

Similarly, by putting $b=\alpha$ into (9), using Eq. (4), $\mathbf{g}^{\alpha j}=0$ and after some calculations, one can deduce Relation (6). This completes the proof.

Proposition 2 The coefficients of conformally doubly warped product Barthel connection for CDWPF are given by

$$
\mathbb{G}_{b}^{a}(\mathrm{x}, \mathrm{y}):=\frac{\partial \mathbb{G}^{a}(\mathrm{x}, \mathrm{y})}{\partial \mathrm{y}^{b}}=\left(\begin{array}{cc}
\mathbb{G}_{j}^{i}(x, u, y, v) & \mathbb{G}_{j}^{\alpha}(x, u, y, v) \\
\mathbb{G}_{\beta}^{i}(x, u, y, v) & \mathbb{G}_{\beta}^{\alpha}(x, u, y, v)
\end{array}\right)
$$

where

$$
\begin{aligned}
\mathbb{G}_{j}^{i}(x, u, y, v):= & \frac{\partial \mathbb{G}^{i}}{\partial y^{j}}=G_{j}^{i}-\frac{1}{4 f_{2}^{2}} \frac{\partial g^{i h}}{\partial y^{j}} \frac{\partial \sigma}{\partial x^{h}}\left(f_{2}^{2} F_{1}^{2}+f_{1}^{2} F_{2}^{2}\right)+\left(\frac{\partial \sigma}{\partial x^{r}} y^{r}+\frac{\partial \sigma}{\partial u^{\alpha}} v^{\alpha}\right) \delta_{j}^{i} \\
& +\frac{\partial \sigma}{\partial x^{j}} y^{i}-\frac{1}{4} g^{i h} \frac{\partial \sigma}{\partial x^{h}} \frac{\partial F_{1}^{2}}{\partial y^{j}}-\frac{1}{4 f_{2}^{2}} \frac{\partial g^{i h}}{\partial y^{j}} \frac{\partial f_{1}^{2}}{\partial x^{h}} F_{2}^{2}+\frac{1}{2 f_{2}^{2}} \frac{\partial f_{2}^{2}}{\partial u^{\alpha}} v^{\alpha} \delta_{j}^{i}, \\
\mathbb{G}_{\beta}^{i}(x, u, y, v):= & \frac{\partial \mathbb{G}^{i}}{\partial v^{\beta}}=\frac{1}{4} g^{i h}\left\{2 \frac{\partial \sigma}{\partial u^{\beta}} \frac{\partial F_{1}^{2}}{\partial y^{h}}-\frac{1}{f_{2}^{2}} f_{1}^{2} \frac{\partial \sigma}{\partial x^{h}} \frac{\partial F_{2}^{2}}{\partial v^{\beta}}+\frac{1}{f_{2}^{2}}\left(\frac{\partial f_{2}^{2}}{\partial u^{\beta}} \frac{\partial F_{1}^{2}}{\partial y^{h}}-\frac{\partial f_{1}^{2}}{\partial x^{h}} \frac{\partial F_{2}^{2}}{\partial \nu^{\beta}}\right)\right\}, \\
\mathbb{G}_{j}^{\alpha}(x, u, y, v):= & \frac{\partial \mathbb{G}^{\alpha}}{\partial y^{j}}=\frac{1}{4} g^{\alpha \gamma}\left\{2 \frac{\partial \sigma}{\partial x^{j}} \frac{\partial F_{2}^{2}}{\partial v^{\gamma}}-\frac{1}{f_{1}^{2}} f_{2}^{2} \frac{\partial \sigma}{\partial u^{\gamma}} \frac{\partial F_{1}^{2}}{\partial y^{j}}+\frac{1}{f_{1}^{2}}\left(\frac{\partial f_{1}^{2}}{\partial x^{j}} \frac{\partial F_{2}^{2}}{\partial v^{\gamma}}-\frac{\partial f_{2}^{2}}{\partial u^{\gamma}} \frac{\partial F_{1}^{2}}{\partial y^{j}}\right)\right\}, \\
\mathbb{G}_{\beta}^{\alpha}(x, u, y, v):= & \frac{\partial \mathbb{G}^{\alpha}}{\partial v^{\beta}}=G_{\beta}^{\alpha}-\frac{1}{4 f_{1}^{2}} \frac{\partial g^{\alpha \gamma}}{\partial v^{\beta}} \frac{\partial \sigma}{\partial u^{\gamma}}\left(f_{2}^{2} F_{1}^{2}+f_{1}^{2} F_{2}^{2}\right)+\left(\frac{\partial \sigma}{\partial x^{r}} y^{r}+\frac{\partial \sigma}{\partial u^{\alpha}} v^{\alpha}\right) \delta_{\beta}^{\alpha} \\
& +\frac{\partial \sigma}{\partial u^{\beta}} v^{\alpha}-\frac{1}{4} g^{\alpha \gamma} \frac{\partial \sigma}{\partial u^{\gamma}} \frac{\partial F_{2}^{2}}{\partial v^{\beta}}-\frac{1}{4 f_{1}^{2}} \frac{\partial g^{\alpha \gamma}}{\partial v^{\beta}} \frac{\partial f_{2}^{2}}{\partial u^{\gamma}} F_{1}^{2}+\frac{1}{2 f_{1}^{2}} \frac{\partial f_{1}^{2}}{\partial x^{r}} y^{r} \delta_{\beta}^{\alpha} .
\end{aligned}
$$

Proof The proof follows from Proposition 1 and taking into account the fact that $\frac{\partial g^{i h}}{\partial y^{j}} \frac{\partial F_{1}^{2}}{\partial y^{h}}=0\left(\frac{\partial g^{\alpha \gamma}}{\partial v^{\beta}} \frac{\partial F_{2}^{2}}{\partial v^{\gamma}}=0\right)$.

Corollary 1 In view of the above proposition and [9], the basis of the vertical and the horizontal distributions $V T M^{o}$ and $H T M^{o} ; M:=M_{1} \times M_{2}$ for the CDWPF $\left(f_{2} M_{1} \times f_{1} M_{2}, F\right)$ 
are given respectively by

$$
\begin{gathered}
\frac{\partial}{\partial \mathrm{y}^{a}}=\frac{\partial}{\partial y^{i}} \delta_{a}^{i}+\frac{\partial}{\partial \nu^{\alpha}} \delta_{a}^{\alpha} \\
\frac{\delta^{d}}{\delta^{d} \mathbf{x}^{a}}=\frac{\delta^{d}}{\delta^{d} x^{i}} \delta_{a}^{i}+\frac{\delta^{d}}{\delta^{d} u^{\alpha}} \delta_{a}^{\alpha},
\end{gathered}
$$

where

$$
\begin{gathered}
\frac{\delta^{d}}{\delta^{d} x^{j}}:=\frac{\partial}{\partial x^{j}}-\mathbb{G}_{j}^{r} \frac{\partial}{\partial y^{j}}-\mathbb{G}_{j}^{\beta} \frac{\partial}{\partial \nu^{\beta}}=\frac{\delta}{\delta x^{j}}-\mathbb{M}_{j}^{r} \frac{\partial}{\partial y^{r}}-\mathbb{G}_{j}^{\beta} \frac{\partial}{\partial \nu^{\beta}}, \\
\frac{\delta^{d}}{\delta^{d} u^{\alpha}}:=\frac{\partial}{\partial u^{\alpha}}-\mathbb{G}_{\alpha}^{r} \frac{\partial}{\partial y^{r}}-\mathbb{G}_{\alpha}^{\mu} \frac{\partial}{\partial v^{\mu}}=\frac{\delta}{\delta u^{\alpha}}-\mathbb{M}_{\alpha}^{\mu} \frac{\partial}{\partial \nu^{\mu}}-\mathbb{G}_{\alpha}^{r} \frac{\partial}{\partial y^{r}},
\end{gathered}
$$

and

$$
\begin{aligned}
\frac{\delta}{\delta x^{j}}:= & \frac{\partial}{\partial x^{j}}-G_{j}^{r} \frac{\partial}{\partial y^{j}}, \frac{\delta}{\delta u^{\alpha}}:=\frac{\partial}{\partial u^{\alpha}}-G_{\alpha}^{\mu} \frac{\partial}{\partial \nu^{\mu}}, \\
\mathbb{M}_{j}^{r}:= & \frac{1}{2 f_{2}^{2}} \frac{\partial f_{2}^{2}}{\partial u^{\alpha}} \nu^{\alpha} \delta_{j}^{r}-\frac{1}{4 f_{2}^{2}} \frac{\partial g^{r} h}{\partial y^{j}} \frac{\partial f_{1}^{2}}{\partial x^{h}} F_{2}^{2}-\frac{1}{4 f_{2}^{2}} \frac{\partial g^{r} h}{\partial y^{j}} \frac{\partial \sigma}{\partial x^{h}}\left(f_{2}^{2} F_{1}^{2}+f_{1}^{2} F_{2}^{2}\right)+\frac{\partial \sigma}{\partial x^{j}} y^{r} \\
& +\left(\frac{\partial \sigma}{\partial x^{t}} y^{t}+\frac{\partial \sigma}{\partial u^{\alpha}} \nu^{\alpha}\right) \delta_{j}^{r}-\frac{1}{4} g^{r h} \frac{\partial \sigma}{\partial x^{h}} \frac{\partial F_{1}^{2}}{\partial y^{j}}, \\
\mathbb{M}_{\alpha}^{\mu}:= & \frac{1}{2 f_{1}^{2}} \frac{\partial f_{1}^{2}}{\partial x^{i}} y^{i} \delta_{\alpha}^{\mu}-\frac{1}{4 f_{1}^{2}} \frac{\partial g^{\mu \lambda}}{\partial \nu^{\alpha}} \frac{\partial f_{2}^{2}}{\partial u^{\lambda}} F_{1}^{2}-\frac{1}{4 f_{1}^{2}} \frac{\partial g^{\mu \lambda}}{\partial \nu^{\alpha}} \frac{\partial \sigma}{\partial u^{\lambda}}\left(f_{2}^{2} F_{1}^{2}+f_{1}^{2} F_{2}^{2}\right)+\frac{\partial \sigma}{\partial x^{j}} y^{r} \\
& +\left(\frac{\partial \sigma}{\partial x^{t}} y^{t}+\frac{\partial \sigma}{\partial u^{\lambda}} \nu^{\lambda}\right) \delta_{\alpha}^{\mu}-\frac{1}{4} g^{\mu \lambda} \frac{\partial \sigma}{\partial u^{\lambda}} \frac{\partial F_{2}^{2}}{\partial \nu^{\alpha}},
\end{aligned}
$$

$\mathbb{G}_{\alpha}^{r}, \mathbb{G}_{j}^{\beta}$ are given by Proposition 2 .

Proposition 3 The coefficients of the conformally doubly warped product Barthel curvature tensor for CDWPF are given by

$$
\mathbb{R}_{a b}^{c}(x, u, y, v):=\frac{\delta^{d} \mathbb{G}_{a}^{c}}{\delta^{d} \mathbf{x}^{b}}-\frac{\delta^{d} \mathbb{G}_{b}^{c}}{\delta^{d} \mathbf{x}^{a}}=\left(\mathbb{R}_{i j}^{k}, \mathbb{R}_{i \beta}^{k}, \mathbb{R}_{\alpha j}^{k}, \mathbb{R}_{\alpha \beta}^{k}, \mathbb{R}_{i j}^{\gamma}, \mathbb{R}_{i \beta}^{\gamma}, \mathbb{R}_{\alpha j}^{\gamma}, \mathbb{R}_{\alpha \beta}^{\gamma}\right),
$$

where

$$
\begin{gathered}
\mathbb{R}_{i j}^{k}:=\frac{\delta^{d} \mathbb{G}_{i}^{k}}{\delta^{d} x^{j}}-\frac{\delta^{d} \mathbb{G}_{j}^{k}}{\delta^{d} x^{i}}=R_{i j}^{k}+\mathfrak{U}_{i j}\left\{\frac{\delta \mathbb{M}_{i}^{k}}{\delta x^{j}}-\mathbb{M}_{j}^{r} G_{i r}^{k}-\mathbb{M}_{j}^{r} \frac{\partial \mathbb{M}_{i}^{k}}{\partial y^{r}}-\mathbb{G}_{j}^{\mu} \frac{\partial \mathbb{M}_{i}^{k}}{\partial \nu^{\mu}}\right\} \\
\mathbb{R}_{i \beta}^{k}:=\frac{\delta^{d} \mathbb{G}_{i}^{k}}{\delta^{d} u^{\beta}}-\frac{\delta^{d} \mathbb{G}_{\beta}^{k}}{\delta^{d} x^{i}}, \quad \mathbb{R}_{\alpha j}^{k}:=\frac{\delta^{d} \mathbb{G}_{\alpha}^{k}}{\delta^{d} x^{j}}-\frac{\delta^{d} \mathbb{G}_{j}^{k}}{\delta^{d} u^{\alpha}}, \\
\mathbb{R}_{\alpha \beta}^{k}:=\frac{\delta^{d} \mathbb{G}_{\alpha}^{k}}{\delta^{d} u^{\beta}}-\frac{\delta^{d} \mathbb{G}_{\beta}^{k}}{\delta^{d} u^{\alpha}}, \quad \mathbb{R}_{i j}^{\gamma}:=\frac{\delta^{d} \mathbb{G}_{i}^{\gamma}}{\delta^{d} x^{j}}-\frac{\delta^{d} \mathbb{G}_{j}^{\gamma}}{\delta^{d} x^{i}}, \\
\mathbb{R}_{i \beta}^{\gamma}:=\frac{\delta^{d} \mathbb{G}_{i}^{\gamma}}{\delta^{d} u^{\beta}}-\frac{\delta^{d} \mathbb{G}_{\beta}^{\gamma}}{\delta^{d} x^{i}}, \quad \mathbb{R}_{\alpha j}^{\gamma}:=\frac{\delta^{d} \mathbb{G}_{\alpha}^{\gamma}}{\delta^{d} x^{j}}-\frac{\delta^{d} \mathbb{G}_{j}^{\gamma}}{\delta^{d} u^{\alpha}}, \\
\mathbb{R}_{\alpha \beta}^{\gamma}:=\frac{\delta^{d} \mathbb{G}_{\alpha}^{\gamma}}{\delta^{d} u^{\beta}}-\frac{\delta^{d} \mathbb{G}_{\beta}^{\gamma}}{\delta^{d} u^{\alpha}}=R_{\alpha \beta}^{\gamma}+\mathfrak{U}_{\alpha \beta}\left\{\frac{\delta \mathbb{M}_{\alpha}^{\gamma}}{\delta u^{\beta}}-\mathbb{M}_{\beta}^{\mu} G_{\alpha \mu}^{\gamma}-\mathbb{M}_{\beta}^{\mu} \frac{\partial \mathbb{M}_{\alpha}^{\gamma}}{\partial \nu^{\mu}}-\mathbb{G}_{\beta}^{r} \frac{\partial \mathbb{M}_{\alpha}^{\gamma}}{\partial y^{r}}\right\} ; \\
\text { conformally doubly warped product Barthel connection given by Proposition } 2 .
\end{gathered}
$$


In view of the above proposition, we have

Corollary 2 If the conformally doubly warped product Finsler manifold $\left(f_{2} M_{1} \times f_{1} M_{2}, F\right)$ is horizontally integrable, then $\left(M_{1}, F_{1}\right)$ and $\left(M_{2}, F_{2}\right)$ are horizontally integrable if and only if the following conditions satisfy

$$
\begin{array}{r}
\mathfrak{U}_{i j}\left\{\frac{\delta \mathbb{M}_{i}^{k}}{\delta x^{j}}-\mathbb{M}_{j}^{r} G_{i r}^{k}-\mathbb{M}_{j}^{r} \frac{\partial \mathbb{M}_{i}^{k}}{\partial y^{r}}-\mathbb{G}_{j}^{\mu} \frac{\partial \mathbb{M}_{i}^{k}}{\partial v^{\mu}}\right\}=0, \\
\mathfrak{U}_{\alpha \beta}\left\{\frac{\delta \mathbb{M}_{\alpha}^{\gamma}}{\delta u^{\beta}}-\mathbb{M}_{\beta}^{\mu} G_{\alpha \mu}^{\gamma}-\mathbb{M}_{\beta}^{\mu} \frac{\partial \mathbb{M}_{\alpha}^{\gamma}}{\partial \nu^{\mu}}-\mathbb{G}_{\beta}^{r} \frac{\partial \mathbb{M}_{\alpha}^{\gamma}}{\partial y^{r}}\right\}=0 .
\end{array}
$$

\section{Berwald connection for CDWPF}

Here, the coefficients of the conformally doubly warped product Berwald connection for CDWPF are studied and investigated.

Proposition 4 The coefficients $\mathbb{G}_{a b}^{c}(x, u, y, v)$ of the conformally doubly warped product Berwald connection for CDWPF are given by

$$
\mathbb{G}_{a b}^{c}(\mathrm{x}, \mathrm{y}):=\frac{\partial \mathbb{G}_{a}^{c}(\mathrm{x}, \mathrm{y})}{\partial \mathrm{y}^{b}}=\left(\mathbb{G}_{i j}^{k}, \mathbb{G}_{i \beta}^{k}, \mathbb{G}_{\alpha j}^{k}, \mathbb{G}_{\alpha \beta}^{k}, \mathbb{G}_{i j}^{\gamma}, \mathbb{G}_{i \beta}^{\gamma}, \mathbb{G}_{\alpha j}^{\gamma}, \mathbb{G}_{\alpha \beta}^{\gamma}\right),
$$

where

$$
\begin{aligned}
& \mathbb{G}_{i j}^{k}:=\frac{\partial \mathbb{G}_{i}^{k}}{\partial y^{j}}=G_{i j}^{k}-\frac{1}{4 f_{2}^{2}} \frac{\partial^{2} g^{k h}}{\partial y^{j} \partial y^{i}} \frac{\partial f_{1}^{2}}{\partial x^{h}} F_{2}^{2}-\frac{1}{4 f_{2}^{2}} \frac{\partial^{2} g^{k h}}{\partial y^{j} \partial y^{i}} \frac{\partial \sigma}{\partial x^{h}}\left(f_{2}^{2} F_{1}^{2}+f_{1}^{2} F_{2}^{2}\right) \\
& -\frac{1}{4} \frac{\partial g^{k h}}{\partial y^{i}} \frac{\partial \sigma}{\partial x^{h}} \frac{\partial F_{1}^{2}}{\partial y^{j}}-\frac{1}{4} \frac{\partial g^{k h}}{\partial y^{j}} \frac{\partial \sigma}{\partial x^{h}} \frac{\partial F_{1}^{2}}{\partial y^{i}}+\frac{\partial \sigma}{\partial x^{i}} \delta_{j}^{k}+\frac{\partial \sigma}{\partial x^{j}} \delta_{i}^{k}-\frac{1}{2} g^{k h} g_{i j} \frac{\partial \sigma}{\partial x^{h}} \\
& =\mathbb{G}_{j i}^{k} \text {, } \\
& \mathbb{G}_{i \beta}^{k}:=\frac{\partial \mathbb{G}_{i}^{k}}{\partial v^{\beta}}=\frac{1}{2 f_{2}^{2}} \frac{\partial f_{2}^{2}}{\partial u^{\beta}} \delta_{i}^{k}-\frac{1}{4 f_{2}^{2}} \frac{\partial g^{k h}}{\partial y^{i}} \frac{\partial f_{1}^{2}}{\partial x^{h}} \frac{\partial F_{2}^{2}}{\partial v^{\beta}}-\frac{f_{1}^{2}}{4 f_{2}^{2}} \frac{\partial g^{k h}}{\partial y^{i}} \frac{\partial \sigma}{\partial x^{h}} \frac{\partial F_{2}^{2}}{\partial v^{\beta}}+\frac{\partial \sigma}{\partial u^{\beta}} \delta_{i}^{k}=\mathbb{G}_{\beta i}^{k}, \\
& \mathbb{G}_{\alpha \beta}^{k}:=\frac{\partial \mathbb{G}_{\alpha}^{k}}{\partial \nu^{\beta}}=-\frac{1}{2 f_{2}^{2}} g^{k h} g_{\alpha \beta}\left\{f_{1}^{2} \frac{\partial \sigma}{\partial x^{h}}+\frac{\partial f_{1}^{2}}{\partial x^{h}}\right\}=\mathbb{G}_{\beta \alpha}^{k}, \\
& \mathbb{G}_{i j}^{\gamma}:=\frac{\partial \mathbb{G}_{i}^{\gamma}}{\partial y^{j}}=-\frac{1}{2 f_{1}^{2}} g^{\gamma \alpha} g_{i j}\left\{f_{2}^{2} \frac{\partial \sigma}{\partial u^{\alpha}}+\frac{\partial f_{2}^{2}}{\partial u^{\alpha}}\right\}=\mathbb{G}_{j i}^{\gamma}, \\
& \mathbb{G}_{i \beta}^{\gamma}:=\frac{\partial \mathbb{G}_{i}^{\gamma}}{\partial v^{\beta}}=\frac{1}{2 f_{1}^{2}} \frac{\partial f_{1}^{2}}{\partial x^{i}} \delta_{\beta}^{\gamma}-\frac{1}{4 f_{1}^{2}} \frac{\partial g^{\gamma \alpha}}{\partial \nu^{\beta}} \frac{\partial f_{2}^{2}}{\partial u^{\alpha}} \frac{\partial F_{1}^{2}}{\partial y^{i}}-\frac{f_{2}^{2}}{4 f_{1}^{2}} \frac{\partial g^{\gamma \alpha}}{\partial \nu^{\beta}} \frac{\partial \sigma}{\partial u^{\alpha}} \frac{\partial F_{1}^{2}}{\partial y^{i}}+\frac{\partial \sigma}{\partial x^{i}} \delta_{\beta}^{\gamma}=\mathbb{G}_{\beta i}^{\gamma}, \\
& \mathbb{G}_{\alpha \beta}^{\gamma}:=\frac{\partial \mathbb{G}_{\alpha}^{\gamma}}{\partial \nu^{\beta}}=G_{\alpha \beta}^{\gamma}-\frac{1}{4 f_{1}^{2}} \frac{\partial^{2} g^{\gamma \lambda}}{\partial v^{\beta} \partial \nu^{\alpha}} \frac{\partial f_{2}^{2}}{\partial u^{\lambda}} F_{1}^{2}-\frac{1}{4 f_{1}^{2}} \frac{\partial^{2} g^{\gamma \lambda}}{\partial \nu^{\beta} \partial v^{\alpha}} \frac{\partial \sigma}{\partial u^{\lambda}}\left(f_{2}^{2} F_{1}^{2}+f_{1}^{2} F_{2}^{2}\right) \\
& -\frac{1}{4} \frac{\partial g^{\gamma \lambda}}{\partial \nu^{\alpha}} \frac{\partial \sigma}{\partial u^{\lambda}} \frac{\partial F_{2}^{2}}{\partial \nu^{\beta}}-\frac{1}{4} \frac{\partial g^{\gamma \lambda}}{\partial \nu^{\beta}} \frac{\partial \sigma}{\partial u^{\lambda}} \frac{\partial F_{2}^{2}}{\partial v^{\alpha}}+\frac{\partial \sigma}{\partial u^{\alpha}} \delta_{\beta}^{\gamma}+\frac{\partial \sigma}{\partial u^{\beta}} \delta_{\alpha}^{\gamma}-\frac{1}{2} g^{\gamma \lambda} g_{\alpha \beta} \frac{\partial \sigma}{\partial u^{\lambda}} \\
& =\mathbb{G}_{\beta \alpha}^{\gamma},
\end{aligned}
$$

and $\left(\mathbb{G}_{j}^{i}, \mathbb{G}_{j}^{\alpha}, \mathbb{G}_{\beta}^{i}, \mathbb{G}_{\beta}^{\alpha}\right)$ are the coefficients of conformally doubly warped product Barthel connection given by Proposition 2.

According to Propositions 2 and 4, we have 
Theorem 1 The conformally doubly warped product Berwald connection for CDWPF is given by

$$
B \Gamma^{d} \equiv\left(\mathbb{G}_{a b}^{c}(x, u, y, v), \mathbb{G}_{b}^{a}(x, u, y, v), 0\right),
$$

where $\mathbb{G}_{b}^{a}$ and $\mathbb{G}_{a b}^{c}$ are respectively given by Propositions 2 and 4.

\section{Cartan connection for CDWPF}

As in the preceding section, the coefficients of the conformally doubly warped product Cartan connection for CDWPF are obtained and studied.

Proposition 5 The coefficients $\bar{\Gamma}_{a b}^{c}(x, u, y, v)$ of the conformally doubly warped product Cartan connection for CDWPF are given by

$$
\bar{\Gamma}_{a b}^{c}(x, u, y, v):=\frac{1}{2} \mathbf{g}^{c e}\left\{\frac{\delta^{d} \mathbf{g}_{e a}}{\delta^{d} \mathbf{x}^{b}}+\frac{\delta^{d} \mathbf{g}_{e b}}{\delta^{d} \mathbf{x}^{a}}-\frac{\delta^{d} \mathbf{g}_{a b}}{\delta^{d} \mathbf{x}^{e}}\right\}=\left(\bar{\Gamma}_{i j}^{k}, \bar{\Gamma}_{i \beta}^{k}, \bar{\Gamma}_{\alpha j}^{k}, \bar{\Gamma}_{\alpha \beta}^{k}, \bar{\Gamma}_{i j}^{\gamma}, \bar{\Gamma}_{i \beta}^{\gamma}, \bar{\Gamma}_{\alpha j}^{\gamma}, \bar{\Gamma}_{\alpha \beta}^{\gamma}\right),
$$

where

$$
\begin{aligned}
& \bar{\Gamma}_{i j}^{k}=\Gamma_{i j}^{k}+\frac{\partial \sigma}{\partial x^{j}} \delta_{i}^{k}+\frac{\partial \sigma}{\partial x^{i}} \delta_{j}^{k}-\frac{\partial \sigma}{\partial x^{h}} g^{k h} g_{i j}-\frac{1}{2} g^{k h}\left\{\mathbb{M}_{j}^{r} \frac{\partial g_{h i}}{\partial y^{r}}+\mathbb{M}_{i}^{r} \frac{\partial g_{h j}}{\partial y^{r}}-\mathbb{M}_{h}^{r} \frac{\partial g_{i j}}{\partial y^{r}}\right\}, \\
& \bar{\Gamma}_{i \beta}^{k}=\frac{1}{2 f_{2}^{2}} g^{k h}\left\{\frac{\partial f_{2}^{2}}{\partial u^{\beta}} g_{h i}-f_{2}^{2} \mathbb{G}_{\beta}^{r} \frac{\partial g_{h i}}{\partial y^{r}}+2 f_{2}^{2} \frac{\partial \sigma}{\partial u^{\beta}} g_{h i}\right\}=\bar{\Gamma}_{\beta i}^{k}, \\
& \bar{\Gamma}_{\alpha \beta}^{k}=-\frac{1}{2 f_{2}^{2}} g^{k h}\left\{\frac{\partial f_{1}^{2}}{\partial x^{h}} g_{\alpha \beta}-f_{1}^{2} \mathbb{G}_{h}^{\lambda} \frac{\partial g_{\alpha \beta}}{\partial v^{\lambda}}+2 f_{1}^{2} \frac{\partial \sigma}{\partial x^{h}} g_{\alpha \beta}\right\}, \\
& \bar{\Gamma}_{i j}^{\gamma}=-\frac{1}{2 f_{1}^{2}} g^{\gamma \lambda}\left\{\frac{\partial f_{2}^{2}}{\partial u^{\lambda}} g_{i j}-f_{2}^{2} \mathbb{G}_{\lambda}^{r} \frac{\partial g_{i j}}{\partial y^{r}}+2 f_{2}^{2} \frac{\partial \sigma}{\partial u^{\lambda}} g_{i j}\right\}, \\
& \bar{\Gamma}_{i \beta}^{\gamma}=\frac{1}{2 f_{1}^{2}} g^{\gamma \lambda}\left\{\frac{\partial f_{1}^{2}}{\partial x^{i}} g_{\lambda \beta}-f_{1}^{2} \mathbb{G}_{i}^{\alpha} \frac{\partial g_{\lambda \beta}}{\partial \nu^{\alpha}}+2 f_{1}^{2} \frac{\partial \sigma}{\partial x^{i}} g_{\lambda \beta}\right\}=\bar{\Gamma}_{\beta i}^{\gamma}, \\
& \bar{\Gamma}_{\alpha \beta}^{\gamma}=\Gamma_{\alpha \beta}^{\gamma}+\frac{\partial \sigma}{\partial u^{\alpha}} \delta_{\beta}^{\gamma}+\frac{\partial \sigma}{\partial u^{\beta}} \delta_{\alpha}^{\gamma}-\frac{\partial \sigma}{\partial u^{\lambda}} g^{\gamma \lambda} g_{\alpha \beta}-\frac{1}{2} g^{\gamma \lambda}\left\{\mathbb{M}_{\alpha}^{\mu} \frac{\partial g_{\lambda \beta}}{\partial v^{\mu}}+\mathbb{M}_{\beta}^{\mu} \frac{\partial g_{\lambda \alpha}}{\partial v^{\mu}}-\mathbb{M}_{\lambda}^{\mu} \frac{\partial g_{\alpha \beta}}{\partial v^{\mu}}\right\} \text {, } \\
& \text { and } \Gamma_{i j}^{k}:=\frac{1}{2} g^{k h}\left\{\frac{\delta g_{h j}}{\delta x^{i}}+\frac{\delta g_{h i}}{\delta x^{i}}-\frac{\delta g_{i j}}{\delta x^{h}}\right\}, \quad \Gamma_{\alpha \beta}^{\gamma}:=\frac{1}{2} g^{\gamma \lambda}\left\{\frac{\delta g_{\lambda \beta}}{\delta u^{\alpha}}+\frac{\delta g_{\lambda \alpha}}{\delta u^{\beta}}-\frac{\delta g_{\alpha \beta}}{\delta u^{\lambda}}\right\}, \mathbb{M}_{j}^{r}, \mathbb{M}_{\alpha}^{\mu} \text { are }
\end{aligned}
$$

Proof The proof follows from the definition of $\bar{\Gamma}_{a b}^{c}(x, u, y, v)$ taking into account Relations (3) and (4) and Corollary 1.

Proposition 6 The coefficients $\bar{C}_{a b}^{c}(x, u, y, v)$ of the conformally doubly warped product Cartan tensor field for CDWPF are given by

$$
\begin{aligned}
\bar{C}_{a b}^{c}(x, u, y, v) & :=\frac{1}{2} \mathbf{g}^{c e} \frac{\partial \mathbf{g}_{a b}}{\partial \mathbf{y}^{e}} \\
& =\left(\bar{C}_{i j}^{k}, \bar{C}_{i \beta}^{k}, \bar{C}_{\alpha j}^{k}, \bar{C}_{\alpha \beta}^{k}, \bar{C}_{i j}^{\gamma}, \bar{C}_{i \beta}^{\gamma}, \bar{C}_{\alpha j}^{\gamma}, \bar{C}_{\alpha \beta}^{\gamma}\right) \\
& =\left(C_{i j}^{k}, 0,0,0,0,0,0, C_{\alpha \beta}^{\gamma}\right),
\end{aligned}
$$

where $C_{i j}^{k}(x, y):=\frac{1}{2} g^{k h} \frac{\partial g_{i j}}{\partial y^{h}}$ and $C_{\alpha \beta}^{\gamma}(u, v):=\frac{1}{2} g^{\gamma \lambda} \frac{\partial g_{\alpha \beta}}{\partial \nu^{\lambda}}$ are the coefficients of Cartan tensor fields of $\left(M_{1}, F_{1}\right)$ and $\left(M_{2}, F_{2}\right)$, respectively. 
Summing up, we have

Theorem 2 The conformally doubly warped product Cartan connection for CDWPF is given by

$$
C \Gamma^{d} \equiv\left(\bar{\Gamma}_{a b}^{c}(x, u, y, v), \mathbb{G}_{b}^{a}(x, u, y, v), \bar{C}_{a b}^{c}(x, u, y, v)\right),
$$

where $\bar{\Gamma}_{a b}^{c}(x, u, y, v)$ and $\bar{C}_{a b}^{c}(x, u, y, v)$ are respectively given by Proposition 5 and 6 , also $\mathbb{G}_{b}^{a}$ is given by Proposition 2.

Corollary 3 For the conformally doubly warped product Finsler manifold CDWPF, we have

(a) the conformally doubly warped product Rund connection $R \Gamma^{d}$ is given by

$$
R \Gamma^{d} \equiv\left(\bar{\Gamma}_{a b}^{c}(x, u, y, v), \mathbb{G}_{b}^{a}(x, u, y, v), 0\right)
$$

(b) the conformally doubly warped product Hasiguchi connection $H \Gamma^{d}$ is given by

$$
H \Gamma^{d} \equiv\left(\mathbb{G}_{a b}^{c}(x, u, y, v), \mathbb{G}_{b}^{a}(x, u, y, v), \bar{C}_{a b}^{c}(x, u, y, v)\right),
$$

where $\mathbb{G}_{b}^{a}(x, u, y, v), \mathbb{G}_{a b}^{c}(x, u, y, v), \bar{\Gamma}_{a b}^{c}(x, u, y, v)$, and $\bar{C}_{a b}^{c}(x, u, y, v)$ are respectively given by Propositions 2, 4, 5 and 6.

\section{Some special Finsler spaces}

In this section, some special Finsler spaces such as Riemannian, C-reducible, and Berwaldian spaces are studied for CDWPF.

First, we begin with the following two lemmas which are useful for this section.

Lemma 1 The coefficients $\bar{I}_{a}(x, u, y, v)$ of the conformally doubly warped product contraction Cartan torsion tensor for CDWPF are given by

$$
\bar{I}_{a}(x, u, y, v):=\mathbf{g}^{b c} \bar{C}_{a b c}(x, u, y, v)=\left(I_{i}(x, y), 0,0,0,0,0,0, I_{\alpha}(u, v)\right)
$$

where $\bar{C}_{a b c}(x, u, y, v):=\mathbf{g}_{d c} \bar{C}_{a b}^{d}, I_{i}(x, y):=g^{j k} C_{i j k}(x, y)$ and $I_{\alpha}(u, v):=g^{\beta \gamma} C_{\alpha \beta \gamma}(u, v)$.

Proof The proof follows from the definition of $\bar{C}_{a b c}(x, u, y, v)$ together with (3), (4) and the fact that $\bar{C}_{a b c}:=\mathbf{g}_{d c} \bar{C}_{a b}^{d}=\left(e^{2 \sigma} f_{2}^{2} C_{i j k}(x, y), 0,0,0,0,0,0, e^{2 \sigma} f_{1}^{2} C_{\alpha \beta \gamma}(u, v)\right)$.

Lemma 2 The coefficients $\hbar_{a b}(x, u, y, v)$ of the conformally doubly warped product angular metric tensor for CDWPF are given by

$$
\hbar_{a b}(x, u, y, v):=\mathbf{g}_{a b}-\frac{1}{F^{2}} \mathrm{y}_{a} \mathrm{y}_{b}=\left(\hbar_{i j}, \hbar_{i \beta}, \hbar_{\alpha j}, \hbar_{\alpha \beta}\right),
$$

where 


$$
\begin{aligned}
& \hbar_{i j}(x, u, y, v):=\mathbf{g}_{i j}-\frac{1}{F^{2}} \mathrm{y}_{i} \mathrm{y}_{j}=e^{2 \sigma} f_{2}^{2}\left(g_{i j}-\frac{f_{2}^{2}}{f_{2}^{2} F_{1}^{2}+f_{1}^{2} F_{2}^{2}} y_{i} y_{j}\right) . \\
& \hbar_{i \beta}(x, u, y, v):=\mathbf{g}_{i \beta}-\frac{1}{F^{2}} \mathrm{y}_{i} \mathrm{y}_{\beta}=-\frac{e^{2 \sigma} f_{1}^{2} f_{2}^{2}}{f_{2}^{2} F_{1}^{2}+f_{1}^{2} F_{2}^{2}} y_{i} v_{\beta} . \\
& \hbar_{\alpha j}(x, u, y, v):=\mathbf{g}_{\alpha j}-\frac{1}{F^{2}} \mathrm{y}_{j} \mathrm{y}_{\alpha}=-\frac{e^{2 \sigma} f_{1}^{2} f_{2}^{2}}{f_{2}^{2} F_{1}^{2}+f_{1}^{2} F_{2}^{2}} y_{j} v_{\alpha} . \\
& \hbar_{\alpha \beta}(x, u, y, v):=\mathbf{g}_{\alpha \beta}-\frac{1}{F^{2}} \mathrm{y}_{\alpha} \mathrm{y}_{\beta}=e^{2 \sigma} f_{1}^{2}\left(g_{\alpha \beta}-\frac{f_{1}^{2}}{f_{2}^{2} F_{1}^{2}+f_{1}^{2} F_{2}^{2}} v_{\alpha} v_{\beta}\right) .
\end{aligned}
$$

Proof The proof follows from the definition of $\hbar_{a b}(x, u, y, v)$ together with (3) and the fact that $\mathrm{y}_{a}:=\mathbf{g}_{a b} \mathrm{y}^{b}=\left(e^{2 \sigma} f_{2}^{2} y_{i}, e^{2 \sigma} f_{2}^{2} v_{\alpha}\right)$.

In view of Lemma 1, we have

Theorem 3 The conformally doubly warped product Finsler manifold $\left(f_{2} M_{1} \times{ }_{f_{1}} M_{2}, F\right)$ is Riemannian if and only if $\left(M_{1}, F_{1}\right)$ and $\left(M_{2}, F_{2}\right)$ are Riemannian manifolds.

A doubly warped product Finsler manifold CDWPF is C-reducible if the associated Matsumoto conformally doubly warped product tensor field $\mathbb{M}_{a b c}(x, u, y, v)$ vanishes identically.

Theorem 4 Every C-reducible conformally doubly warped product Finsler manifold $\left(f_{2} M_{1} \times{ }_{f_{1}} M_{2}, F\right)$ is Riemannian.

Proof The Matsumoto conformally doubly warped product tensor field $\mathbb{M}_{a b c}(x, u, y, v)$ is defined by

$$
\mathbb{M}_{a b c}(x, u, y, v):=\bar{C}_{a b c}-\frac{1}{n+1}\left\{\bar{I}_{a} \hbar_{b c}+\bar{I}_{b} \hbar_{c a}+\bar{I}_{c} \hbar_{a b}\right\}
$$

Hence, using Lemmas 1 and 2, the component $\mathbb{M}_{\alpha j k}(x, u, y, v)$ has the form

$$
\mathbb{M}_{\alpha j k}(x, u, y, v)=\frac{1}{n+1} \frac{e^{2 \sigma} f_{1}^{2} f_{2}^{2} v_{\alpha}}{f_{2}^{2} F_{1}^{2}+f_{1}^{2} F_{2}^{2}}\left(I_{j} y_{k}+I_{k} y_{j}\right)-\frac{e^{2 \sigma} f_{2}^{2} I_{\alpha}}{(n+1)}\left(g_{j k}-\frac{f_{2}^{2}}{f_{2}^{2} F_{1}^{2}+f_{1}^{2} F_{2}^{2}} y_{j} y_{k}\right) .
$$

Consequently, one can show that

$$
\mathbb{M}_{\alpha j k}(x, u, y, v) y_{j} y_{k}=-\frac{e^{2 \sigma} f_{2}^{2} F_{1}^{2} I_{\alpha}}{(n+1)}\left(1-\frac{f_{2}^{2} F_{1}^{2}}{f_{2}^{2} F_{1}^{2}+f_{1}^{2} F_{2}^{2}}\right) .
$$

Now, if $\mathbb{M}_{\alpha j k}(x, u, y, v)$ vanishes, then $I_{\alpha}$ vanishes. This means that $\left(M_{2}, F_{2}\right)$ is Riemannian. Similarly, if $\mathbb{M}_{i \alpha \beta}(x, u, y, v)=0$, then $I_{i}=0$. Hence, $\left(M_{1}, F_{1}\right)$ is also Riemannian. Therefore, using Theorem 3 , the result follows.

In view of Proposition 4, we have

Proposition 7 The coefficients $\mathbb{B}_{a b c}^{d}(x, u, y, v)$ of the conformally doubly warped product Berwald curvature tensor for CDWPF are given by 


$$
\mathbb{B}_{a b c}^{d}(x, u, y, v):=\frac{\partial \mathbb{G}_{a b}^{d}}{\partial \mathrm{y}^{c}}=\left(\mathbb{B}_{i j l}^{k}, \mathbb{B}_{i \beta l}^{k}, \mathbb{B}_{\alpha \beta l}^{k}, \mathbb{B}_{\alpha \beta \lambda}^{k}, \mathbb{B}_{i j l}^{\gamma}, \mathbb{B}_{i \beta l}^{\gamma}, \mathbb{B}_{\alpha \beta l}^{\gamma}, \mathbb{B}_{\alpha \beta \lambda}^{\gamma}\right),
$$

where

$$
\begin{aligned}
& \mathbb{B}_{i j l}^{k}:=\frac{\partial \mathbb{G}_{i j}^{k}}{\partial y^{l}}=B_{i j l}^{k}-\frac{1}{4 f_{2}^{2}} \frac{\partial^{3} g^{k h}}{\partial y^{l} \partial y^{j} \partial y^{i}} \frac{\partial f_{1}^{2}}{\partial x^{h}} F_{2}^{2}-\frac{1}{4 f_{2}^{2}} \frac{\partial^{3} g^{k h}}{\partial y^{l} \partial y^{j} \partial y^{i}} \frac{\partial \sigma}{\partial x^{h}}\left(f_{2}^{2} F_{1}^{2}+f_{1}^{2} F_{2}^{2}\right) \\
& -\frac{1}{4} \frac{\partial^{2} g^{k h}}{\partial y^{j} \partial y^{i}} \frac{\partial F_{1}^{2}}{\partial y^{l}} \frac{\partial \sigma}{\partial x^{h}}-\frac{1}{4} \frac{\partial^{2} g^{k h}}{\partial y^{l} \partial y^{i}} \frac{\partial \sigma}{\partial x^{h}} \frac{\partial F_{1}^{2}}{\partial y^{j}}-\frac{1}{2} \frac{\partial g^{k h}}{\partial y^{i}} \frac{\partial \sigma}{\partial x^{h}} g_{j l}-\frac{1}{4} \frac{\partial^{2} g^{k h}}{\partial y^{l} \partial y^{j}} \frac{\partial \sigma}{\partial x^{h}} \frac{\partial F_{1}^{2}}{\partial y^{i}} \\
& -\frac{1}{2} \frac{\partial g^{k h}}{\partial y^{j}} \frac{\partial \sigma}{\partial x^{h}} g_{i l}-\frac{1}{2} \frac{\partial g^{k h}}{\partial y^{l}} \frac{\partial \sigma}{\partial x^{h}} g_{i j}-g^{k h} \frac{\partial \sigma}{\partial x^{h}} C_{i j l}, \\
& \mathbb{B}_{i \beta l}^{k}:=\frac{\partial \mathbb{G}_{i \beta}^{k}}{\partial y^{l}}=-\frac{1}{4 f_{2}^{2}} \frac{\partial^{2} g^{k h}}{\partial y^{l} \partial y^{i}} \frac{\partial F_{2}^{2}}{\partial \nu^{\beta}}\left\{\frac{\partial f_{1}^{2}}{\partial x^{h}}+f_{1}^{2} \frac{\partial \sigma}{\partial x^{h}}\right\}, \\
& \mathbb{B}_{\alpha \beta l}^{k}:=\frac{\partial \mathbb{G}_{\alpha \beta}^{k}}{\partial y^{l}}=-\frac{1}{2 f_{2}^{2}} \frac{\partial g^{k h}}{\partial y^{l}} g_{\alpha \beta}\left\{\frac{\partial f_{1}^{2}}{\partial x^{h}}+f_{1}^{2} \frac{\partial \sigma}{\partial x^{h}}\right\} \\
& \mathbb{B}_{\alpha \beta \lambda}^{k}:=\frac{\partial \mathbb{G}_{\alpha \beta}^{k}}{\partial \nu^{\lambda}}=-\frac{1}{f_{2}^{2}} g^{k h} C_{\alpha \beta \lambda}\left\{\frac{\partial f_{1}^{2}}{\partial x^{h}}+f_{1}^{2} \frac{\partial \sigma}{\partial x^{h}}\right\}, \\
& \mathbb{B}_{i j l}^{\gamma}:=\frac{\partial \mathbb{G}_{i j}^{\gamma}}{\partial y^{l}}=-\frac{1}{f_{1}^{2}} g^{\gamma \mu} C_{i j l}\left\{\frac{\partial f_{2}^{2}}{\partial u^{\mu}}+f_{2}^{2} \frac{\partial \sigma}{\partial u^{\mu}}\right\}, \\
& \mathbb{B}_{i \beta l}^{\gamma}:=\frac{\partial \mathbb{G}_{i \beta}^{\gamma}}{\partial y^{l}}=-\frac{1}{2 f_{1}^{2}} \frac{\partial g^{\gamma \mu}}{\partial v^{\beta}} g_{i l}\left\{\frac{\partial f_{2}^{2}}{\partial u^{\mu}}+f_{2}^{2} \frac{\partial \sigma}{\partial u^{\mu}}\right\}, \\
& \mathbb{B}_{\alpha \beta l}^{\gamma}:=\frac{\partial \mathbb{G}_{\alpha \beta}^{\gamma}}{\partial y^{l}}=-\frac{1}{4 f_{1}^{2}} \frac{\partial^{2} g^{\gamma \mu}}{\partial \nu^{\beta} \partial v^{\alpha}} \frac{\partial F_{1}^{2}}{\partial y^{l}}\left\{\frac{\partial f_{2}^{2}}{\partial u^{\mu}}+f_{2}^{2} \frac{\partial \sigma}{\partial u^{\mu}}\right\}, \\
& \mathbb{B}_{\alpha \beta \lambda}^{\gamma}:=\frac{\partial \mathbb{G}_{\alpha \beta}^{\gamma}}{\partial \nu^{\lambda}}=B_{\alpha \beta \lambda}^{\gamma}-\frac{1}{4 f_{1}^{2}} \frac{\partial^{3} g^{\gamma \mu}}{\partial \nu^{\lambda} \partial \nu^{\beta} \partial \nu^{\alpha}} \frac{\partial f_{2}^{2}}{\partial u^{\mu}} F_{1}^{2}-\frac{1}{4 f_{1}^{2}} \frac{\partial^{3} g^{\gamma \mu}}{\partial \nu^{\lambda} \partial v^{\beta} \partial \nu^{\alpha}} \frac{\partial \sigma}{\partial u^{\mu}}\left(f_{2}^{2} F_{1}^{2}+f_{1}^{2} F_{2}^{2}\right) \\
& -\frac{1}{4} \frac{\partial^{2} g^{\gamma \mu}}{\partial \nu^{\beta} \partial \nu^{\alpha}} \frac{\partial F_{2}^{2}}{\partial v^{\lambda}} \frac{\partial \sigma}{\partial u^{\mu}}-\frac{1}{4} \frac{\partial^{2} g^{\gamma \mu}}{\partial v^{\lambda} \partial \nu^{\alpha}} \frac{\partial \sigma}{\partial u^{\mu}} \frac{\partial F_{2}^{2}}{\partial v^{\beta}}-\frac{1}{2} \frac{\partial g^{\gamma \mu}}{\partial \nu^{\alpha}} \frac{\partial \sigma}{\partial u^{\mu}} g_{\beta \lambda}-g^{\gamma \mu} \frac{\partial \sigma}{\partial u^{\mu}} C_{\alpha \beta \lambda} \\
& -\frac{1}{2} \frac{\partial g^{\gamma \mu}}{\partial v^{\beta}} \frac{\partial \sigma}{\partial u^{\mu}} g_{\alpha \lambda}-\frac{1}{2} \frac{\partial g^{\gamma \mu}}{\partial v^{\lambda}} \frac{\partial \sigma}{\partial u^{\mu}} g_{\alpha \beta}-\frac{1}{4} \frac{\partial^{2} g^{\gamma \mu}}{\partial v^{\lambda} \partial v^{\beta}} \frac{\partial \sigma}{\partial u^{\mu}} \frac{\partial F_{2}^{2}}{\partial v^{\alpha}},
\end{aligned}
$$

and $\mathbb{G}_{i j}^{k}, \mathbb{G}_{i \beta}^{k}, \mathbb{G}_{\alpha \beta}^{k}, \mathbb{G}_{i j}^{\gamma}, \mathbb{G}_{i \beta}^{\gamma}, \mathbb{G}_{\alpha \beta}^{\gamma}$ are the coefficients of conformally doubly warped product Berwald connection given by Proposition 4.

Definition 3 A conformally doubly warped product Finsler manifold $\left(f_{2} M_{1} \times{ }_{f_{1}} M_{2}, F\right)$ satisfying the following conditions: $\frac{\partial f_{1}^{2}}{\partial x^{h}}+f_{1}^{2} \frac{\partial \sigma}{\partial x^{h}} \neq 0$ and $\frac{\partial f_{2}^{2}}{\partial u^{\mu}}+f_{2}^{2} \frac{\partial \sigma}{\partial u^{\mu}} \neq 0$ is called a conditionally conformally doubly warped product Finsler manifold.

Theorem 5 Every conditionally conformally doubly warped product Finsler manifold $\left(f_{2} M_{1} \times f_{1} M_{2}, F\right)$ with vanishing Berwald curvature is Riemannian.

Definition 4 A conformally doubly warped product Finsler manifold $\left(f_{2} M_{1} \times{ }_{f_{1}} M_{2}, F\right)$ with constant conformal factor $\sigma$ is called a homothety doubly warped product Finsler manifold. 
A Finsler manifold is called Berwald if its hv-Berwald curvature tensor vanishes identically.

Theorem 6 Let $\left(f_{2} M_{1} \times{ }_{f_{1}} M_{2}, F\right)$ be a homothety doubly warped product Finsler manifold and $f_{1}$ is constant on $M_{1}\left(f_{2}\right.$ is constant on $\left.M_{2}\right)$. Then, $\left(f_{2} M_{1} \times f_{1} M_{2}, F\right)$ is Berwaldian if and only if $\left(M_{1}, F_{1}\right)$ is Riemannian, $\left(M_{2}, F_{2}\right)$ is Berwaldian and $\frac{\partial g^{\alpha \gamma}}{\partial v^{\lambda}} \frac{\partial f_{2}^{2}}{\partial u^{\alpha}}=0\left(\left(M_{2}, F_{2}\right)\right.$ is Riemannian, $\left(M_{1}, F_{1}\right)$ is Berwaldian and $\left.\frac{\partial g^{i j}}{\partial y^{l}} \frac{\partial f_{1}^{2}}{\partial u^{i}}=0\right)$

Proof Suppose that $\left(f_{2} M_{1} \times f_{1} M_{2}, F\right)$ is a homothety doubly warped product Finsler manifold and $f_{1}$ is constant on $M_{1}$. Then, from Proposition 7, one can show that

$$
\begin{aligned}
\mathbb{B}_{i j l}^{\gamma} & =-\frac{1}{f_{1}^{2}} g^{\gamma \mu} C_{i j l} \frac{\partial f_{2}^{2}}{\partial u^{\mu}}, \\
\mathbb{B}_{i \beta l}^{\gamma} & =-\frac{1}{2 f_{1}^{2}} \frac{\partial g^{\gamma \mu}}{\partial v^{\beta}} g_{i l} \frac{\partial f_{2}^{2}}{\partial u^{\mu}}, \\
\mathbb{B}_{\alpha \beta \lambda}^{\gamma} & =B_{\alpha \beta \lambda}^{\gamma}-\frac{1}{4 f_{1}^{2}} \frac{\partial^{3} g^{\gamma \mu}}{\partial v^{\lambda} \partial v^{\beta} \partial v^{\alpha}} \frac{\partial f_{2}^{2}}{\partial u^{\mu}} F_{1}^{2} .
\end{aligned}
$$

Now, if ( $\left.f_{2} M_{1} \times f_{1} M_{2}, F\right)$ is Berwaldian, then using (12), we conclude that $C_{i j k}$ vanishes, and hence $\left(M_{1}, F_{1}\right)$ is Riemanian. On the other hand, from (13), we get $\frac{\partial g^{\gamma \mu}}{\partial \nu^{\beta}} \frac{\partial f_{2}^{2}}{\partial u^{\mu}}=0$. Consequently, $\frac{\partial^{3} g^{\gamma \mu}}{\partial v^{\lambda} \partial v^{\beta} \partial v^{\alpha}} \frac{\partial f_{2}^{2}}{\partial u^{\mu}}=0$. From which together with (14), we get $B_{\alpha \beta \lambda}^{\gamma}=0$. This means that $\left(M_{2}, F_{2}\right)$ is Berwaldian. The converse is proved by the same manner. This completes the proof.

\section{Concluding remarks}

1. In this paper, we obtained some results concerning the conformally doubly warped product Finsler manifold CDWPF; namely, we got formulas for the following:

(a) Canonical spray, Barthel connection and its curvature tensor (Propositions 1, 2, and 3) are calculated.

(b) Berwald and Cartan connections (Theorems 1 and 2) are computed.

(c) Some special Finsler spaces such as Riemannian, C-reducible and Berwald spaces (Theorems 3, 4 and 6) are studied.

2. The above results can be obtained for the conformally warped product Finsler manifold CWPF by setting $f_{1}=1$.

3. The same results can be achieved for the doubly warped product Finsler manifold DWPF by setting $\sigma=0$ which was investigated by Peyghan and Tayebi [9] .

4. The mentioned results above can be obtained for the warped product Finsler manifold WPF by setting $\sigma=0, f_{1}=1$, and $f_{2}=1$.

Abbreviations

CDWPF: Conformally doubly warped product Finsler manifolds; CPF: Conformally product Finsler manifolds; CWPF:

Conformally warped product Finsler manifolds; DWPF: Doubly warped product Finsler manifolds

\section{Acknowledgments}

The authors are grateful to the referees, Professor Nabil L. Youssef, and Doctor S. G. Elgendi for their valuable suggestions and comments. 


\section{Funding}

Not applicable

Availability of data and materials

Not applicable

\section{Competing interests}

The authors declare that they have no competing interests.

Received: 12 July 2019 Accepted: 28 November 2019

Published online: 23 December 2019

\section{References}

1. Allison, D. E.: Pseudoconvexity in Lorentzian doubly warped product. Geom. Dedicata. 39, 223-227 (1991)

2. Beem, J. K., Ehrlich, P., Powell, T. G.: Warped product manifolds in relativity, in Selected Studies, North-Holland Pub. Co., Amsterdam (1982)

3. Beem, J. B., Powell, T. G.: Geodesic completeness and maximality in Lorentzian warped products. Tensor, N.S. 39 31-36 (1982)

4. Gebarowski, A.: On conformally recurrent doubly warped products. Tensor, N.S. 57, 192-196 (1996)

5. Unal, B.: Doubly warped products. Diff. Geom. Appl. 15, 253-263 (2001)

6. Asanov, G. S.: Finslerian extensions of Schwarzschild metric. Fortschr. Phys. 40, 667-693 (1992)

7. Asanov, G. S.: Finslerian metric functions over the product $R \times M$ and their potential applications. Rep. Math. Phys. 41, 117-132 (1998)

8. Shen, Z:: Finsler manifolds of constant positive curvature. Contemp. Math. 196, 83-93 (1995)

9. Peyghan, E., Tayebi, A.: On doubly warped prouduct Finsler manifolds. J. Nonlinear Anal. Real World Appl. 13, 1703-1720 (2012). arXiv: 1110.6830v1 [math.DG]

10. Bao, D., Chern, S. S., Shen, Z.: An Introduction to Riemannian Finsler Geometry. Spinger-Verlag, New York (2000)

11. Matsumoto, M.: The theory of Finsler connections, Publication of the study group of geometry, 5. Okayama University, Japan (1970)

12. Rund, H.: The differential geometry of Finsler spaces. Springer-Verlag, Berlin (1959)

13. Shen, Z:: Differential Geometry of Spray and Finsler Spaces. Kluwer Academic Publishers (2001). https://doi.org/10. 1007/978-94-015-9727-2

14. Tayebi, A., Naja, B.: Shen's process on Finslerian connections. Bull. Iran. Math. Soc. 36, 57-73 (2010)

15. Matsumoto, M.: On C-reducible Finsler spaces. Tensor, N.S. 24, 29-37 (1972)

\section{Publisher's Note}

Springer Nature remains neutral with regard to jurisdictional claims in published maps and institutional affiliations.

\section{Submit your manuscript to a SpringerOpen ${ }^{\circ}$ journal and benefit from:}

- Convenient online submission

- Rigorous peer review

Open access: articles freely available online

High visibility within the field

- Retaining the copyright to your article

Submit your next manuscript at $\triangleright$ springeropen.com 